\title{
Single bubble deformation and breakup in simple shear flow
}

\author{
Nadina Müller-Fischer · Philip Tobler • \\ Marco Dressler · Peter Fischer · Erich J. Windhab
}

Published online: 4 August 2009

(C) Springer-Verlag 2009

\section{Erratum to: Exp Fluids (2008) 45:917-926 DOI 10.1007/s00348-008-0509-1}

Unfortunately, a published article contains error in the bubble dimension data. The modified analysis for the error was given below.

In the publication 'Single bubble deformation and breakup in simple shear flow' (2008) we report on the bubble deformation and bubble breakup in simple shear flow. The obtained results were compared to the previous findings by Canedo et al. (1993) and Rust and Manga (2002) (Figure 6 of Müller-Fischer et al. 2008). The deviations of the deformation parameters $D$ and $L / x_{B}$ between our results and those by Canedo et al. and Rust and Manga were explained by 'higher curvature effects due to confinement and bound flow conditions, limitation in image analysis, the influence of surfactants, and limited validity of the used models'. Revisiting the original data files, however, leads to the conclusion that we used for the calculation of the bubble deformation the diameter of the bubble and not the bubble radius. As a consequence, all

The online version of the original article can be found under doi:10.1007/s00348-008-0509-1.

N. Müller-Fischer · P. Tobler · M. Dressler · P. Fischer $(\bowtie)$

E. J. Windhab

Institute of Food Science \& Nutrition, ETH Zurich,

8092 Zurich, Switzerland

e-mail: peter.fischer@ilw.agrl.ethz.ch

N. Müller-Fischer

Bühler AG, 9240 Uzwil, Switzerland

e-mail: nadina.mueller-fischer@buhlergroup.com deformation data are corrupted by the factor of two. Equations 5 and 10 in (2008) have to be rewritten as:

$\frac{L}{a} \cong 3.45 \cdot C a^{0.5}$

$\frac{L}{a}=3.1 \cdot C a^{0.43}$

with $a$ as bubble radius (instead of $x_{B}$ as bubble diameter). Using the correct bubble dimensions Figure 6 of MüllerFischer et al. (2008) can be redrawn as shown in Fig. 1 showing the deformation parameter parameter $L / a$ as a function of the Capillary number $C a$.

Both models by Hinch and Acrivos (1980) (Eq. 1) and Canedo et al. (1993) (Eq. 2) are used to describe the measured data. The model of Hinch and Acrivos is fitting the experimental data well for $\mathrm{Ca} \leq 20$ but fail at large bubble deformations. The same result has been indicated in

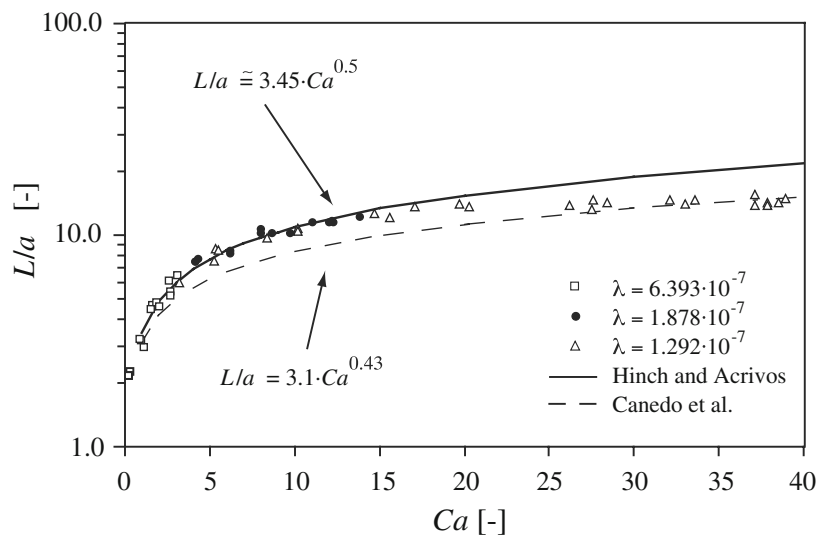

Fig. 1 Deformation parameter $L / a$ as a function of the Capillary number $\mathrm{Ca}$ for bubbles in simple shear. Symbols represent measured values, the solid line represents the model prediction by Hinch and Acrivos (1980), the dashed line the model for Canedo et al. (1993) 
Rust and Manga (2002). On the other hand, i.e., above $C a \geq 25$, Eq. 2 by Canedo et al. (1993) can match the experiment results well. The validity of both models in the different $\mathrm{Ca}$ number regime is related to the assumptions made. Hinch and Acrivos (1980) take only circular bubble cross-section into account, which is not correct for large bubble deformations as shown by Canedo et al. (1993). The latter authors assume an ellipsoidal cross-section, which fit the high deformation regime of our bubble deformation experiments well. In conclusion, Eq. 1 can be used to describe the bubble deformation for Capillary numbers smaller than 20 and Eq. 2 can be used when the Capillary numbers are larger than $25(\lambda \ll 1$ and $R e \ll 1)$.

Acknowledgments The authors would like to thank Malin Liu, Department of Chemical Engineering, Tsinghua University, Beijing
100084, China for pointing out the mistake in the droplet dimension analysis.

\section{References}

Canedo E, Favelukis M, Tadmor Z, Talmon Y (1993) An experimental study of bubble deformation in viscous liquids in simple shear flow. AIChE J 39:553

Hinch E, Acrivos A (1980) Long slender drops in a simple shear flow. J Fluid Mech 98:305

Müller-Fischer N, Tobler P, Dressler M, Fischer P, Windhab E (2008) Single bubble deformation and breakup in simple shear flow. Exp Fluids 45:917

Rust A, Manga M (2002) Bubble shapes and orientation in low Re simple shear flow. J Colloid Interface Sci 249:476 